\title{
Observations on the sensory nature of the intramuscular nerve action potential
}

\author{
A. FIASCHI \\ From the Institute of the Clinic for Nervous and Mental Diseases, \\ University of Padua, Verona, Italy
}

SUMMARY The nature and the origin of the small pre-potential wave which can be recorded immediately before the main muscle action potential on indirect stimulation was studied in the median nerve of 12 normal subjects. It is considered to be a sensory antidromic response recorded from the large afferent fibres which innervate the thumb because it was recorded in all 12 subjects, and the threshold was always below the motor threshold. An antidromic response was recorded with stimulating electrodes at the thumb and recording electrodes at the palm. Both antidromic and orthodromic responses were recorded with stimulating electrodes at the palm. The amplitude of the pre-potential was higher at the recording point closest to the sensory fibres for the thumb and progressively decreased with distance in the other points.

The measurement of motor conduction velocity can sometimes be difficult because of some possible errors in the latency measurements. One of these has been described by Simpson (1964) as an initial small negative wave recorded before the muscle action potential, the origin of which is not completely clear.

Simpson (1964) suggested that it can be considered a nerve action potential in the terminal fibres. Buchthal and Rosenfalck (1966) theorized that this potential could either originate from motor fibres or be an antidromic sensory response. In favour of the former hypothesis is the fact that it usually appeared with the same threshold as the motor response.

Gutmann (1969) studied this pre-potential wave in normal median nerve and in median nerve in which there has been a selective loss of motor axons. He concluded that the prepotential is mainly derived from sensory axons conducting antidromically; however, motor axons may also contribute.

The purpose of this study is to investigate the origin of this initial pre-potential wave (Fig. 1).

\section{METHODS}

The examinations were performed on 12 subjects ranging from 20 to 40 years of age without signs or symptoms of neuromuscular disorders.

The stimulating electrodes were two surface electrodes placed at the wrist $25 \mathrm{~mm}$ apart along the nerve or at the palm. (We also used stainless steel needles $0.6 \mathrm{~mm}$ in diameter completely insulated, except at the tip, but no differences were noted.)

As recording electrodes, we used three concentric needles $0.45 \mathrm{~mm}$ in diameter with a leading-off area of $0.03 \mathrm{~mm}^{2}$. One of these was placed on the thenar muscles, another close to the sensory fibres of the thumb on the palm, and another between these two electrodes.

Two surface electrodes were also placed around the thumb with the anode on the distal part of the distal phalanx and the cathode $10-20 \mathrm{~mm}$ proximal to it in order to record the sensory antidromic response (Fig. 2).

The stimulus was always a $(0.2 \mathrm{msec})$ rectangular electrical pulse.

During all investigations the stimulating current was always less than $100 \mathrm{v}$.

Single oscilloscope sweeps and superimposed sweeps of about seven waves were photographed with a time base of $0 \cdot 5-1-2 \mathrm{msec} / \mathrm{cm}$.

The electromyographic amplification was always adjusted to give a peak deflection of at least $10 \mathrm{~mm}$ on the oscilloscope tube.

The latency of the evoked potential was measured from the onset of the stimulus to the peak of the 


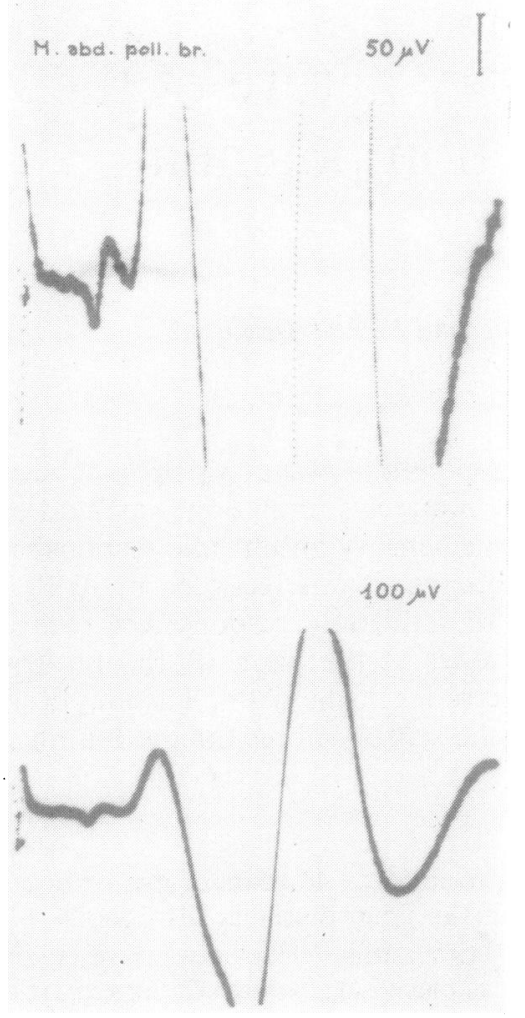

first positive deflection; the amplitude was measured peak-to-peak; the distances were measured betw\&en the more distal electrode at wrist (cathode) and ghe concentric needles, and from these to the cathodesof the surface electrodes placed around the thumb.

The temperature of the limb throughout The investigation was $34^{\circ}-36^{\circ} \mathrm{C}$.

\section{RESULTS}

With high amplification in all 12 subjects, recorded a pre-potential wave preceding main deflection of the muscle action potent The threshold of this first response was c ․ㅠstantly below the motor threshold, even when elicited with the stimulating current at half value necessary to obtain the motor threshold (Fig. 9).

Stimulation of the median nerve at the wijst evoked a potential with the same latency for three recording electrodes, being registered wh a mean value of $1.7 \pm 0.2 \mathrm{msec}$ at a conduction

FIG. 1. Initial pre-potential wave recorded before the main muscle action potential. Stimulation at the wrist (sweep 2).

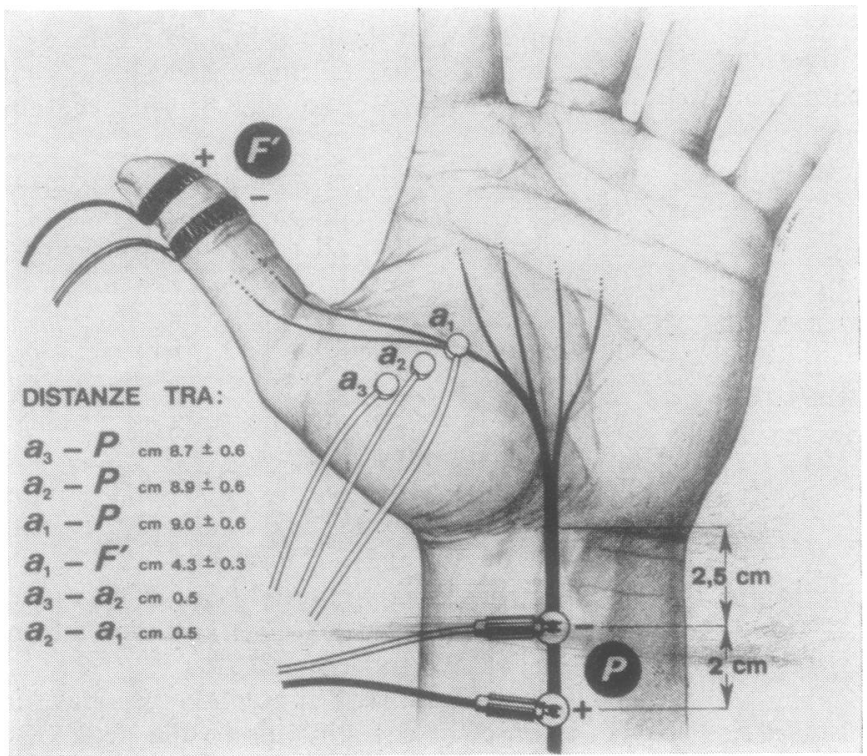

FIG. 2. Position of the recording and stimulating electrodes. 


\section{$50 \mu \mathrm{VI}$}
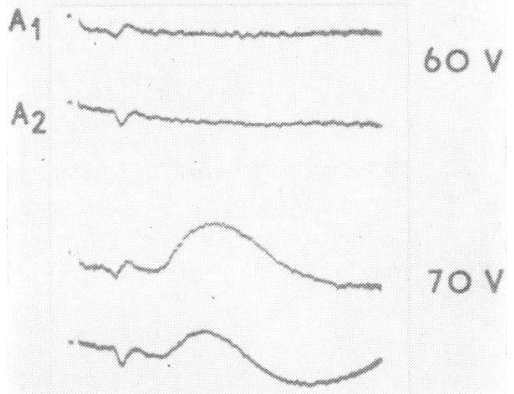

$80 \mathrm{~V}$
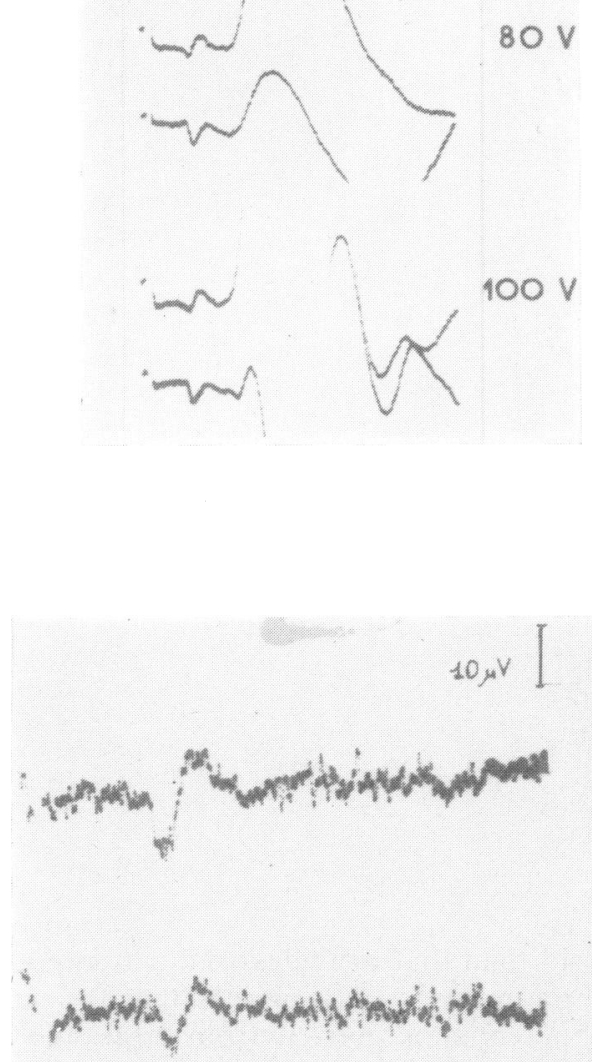

$\mathrm{A}_{2}$

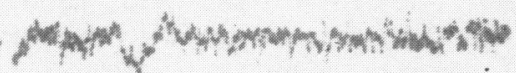

velocity of $48 \pm 0 \cdot 3 \mathrm{~m} / \mathrm{sec}$ (Fig. 4). The amplitude was not constant for all recording electrodes, but it was highest at the recording point closest to the sensory fibres and progressively decreased at the other points.

The mean amplitude in different subjects varied with respect to the three different electrodes ranging from $30 \mu \mathrm{V}$ to $10 \mu \mathrm{V}$, from $20 \mu \mathrm{V}$ to $8 \mu \mathrm{V}$, and from $15 \mu \mathrm{V}$ to $5 \mu \mathrm{V}$.

With the same intensity of stimulating current, a sensory antidromic response was recorded in the thumb, and by stimulating the sensory fibres of the thumb, a potential was evoked in all three recording electrodes with the same latency and with the same decrease in amplitude (Figs 5 and 6). Moreover, an antidromic potential at the thumb and an orthodromic potential at the wrist were obtained at the same time by stimulation of the sensory fibres at the palm (Fig. 7).

Finally, it must be remembered that the amplitude of this pre-potential increases until it

FIG. 3. Stimulation at wrist. The threshold of the intramuscular nerve action potential was constantly below the motor threshold (sweep 2).

FIG. 4. Stimulation of the median nerve at the wrist evoked a potential with the same latency for the three recording electrodes and the amplitude was higher at the recording point closer to the sensory fibres (sweep 1). 


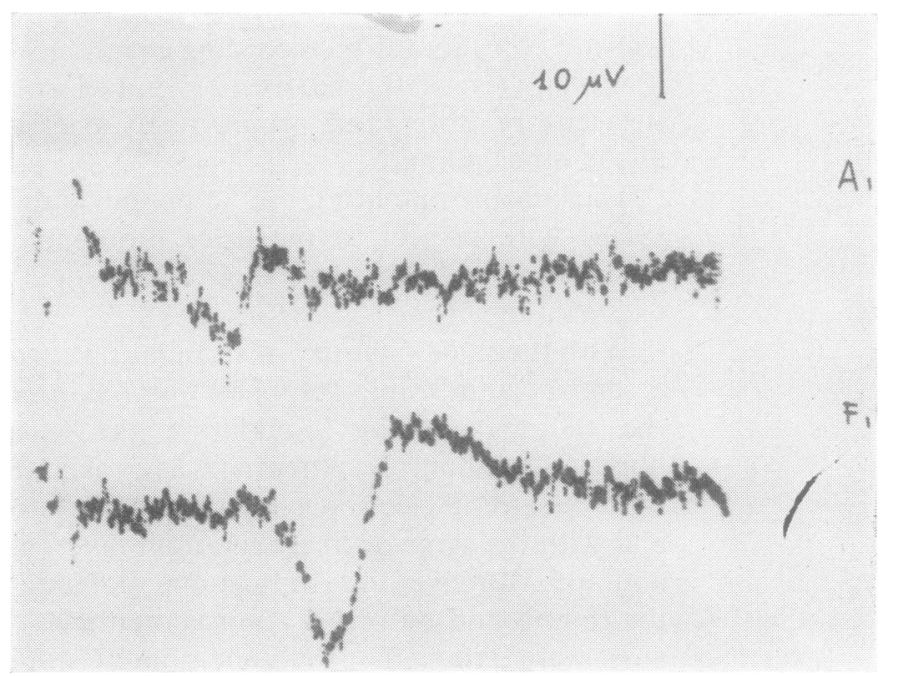

FIG. 5. Stimulation of the median nerve at the wrist evoked a sensory antidromic response at the palm (A) and at the thumb (sweep 1).

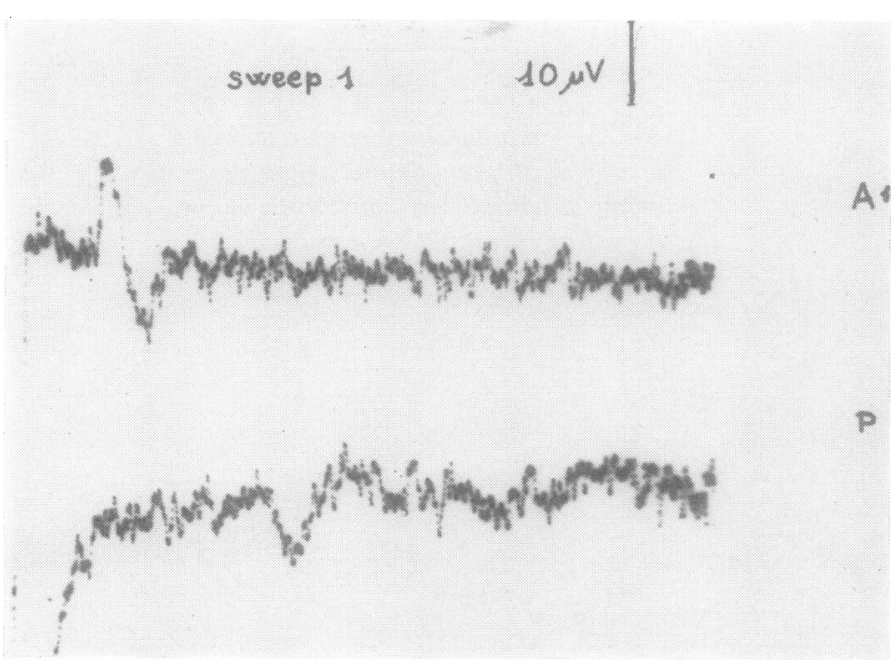

FIG. 6. With the same intensity of stimulating current at the thumb a ô. sensory orthodromic response was evoked at the palm (A) and at the wrist.

reaches a maximum value with a stimulus which may exceed the motor threshold.

\section{DISCUSSION}

These studies strongly suggest that the prepotential wave which may be recorded before the main action potential is a sensory antidromic response.

Our opinion is based on these points: (1) The potential was recorded in all subjects at a lower threshold than that of the evoked action poten tial of the muscle. This suggests that the nerve action potential is derived from large afferent nerve fibres with faster conduction velocity that the motor fibres. (2) Moreover, an antidromio sensory response with the stimulating electrodes at the thumb was always recorded and, at the same time, an orthodromic and antidromic sensory response was recorded with the stimulating electrodes at the palm. (3) For these reasons we agree with Gutmann (1969) and with Lamberto 


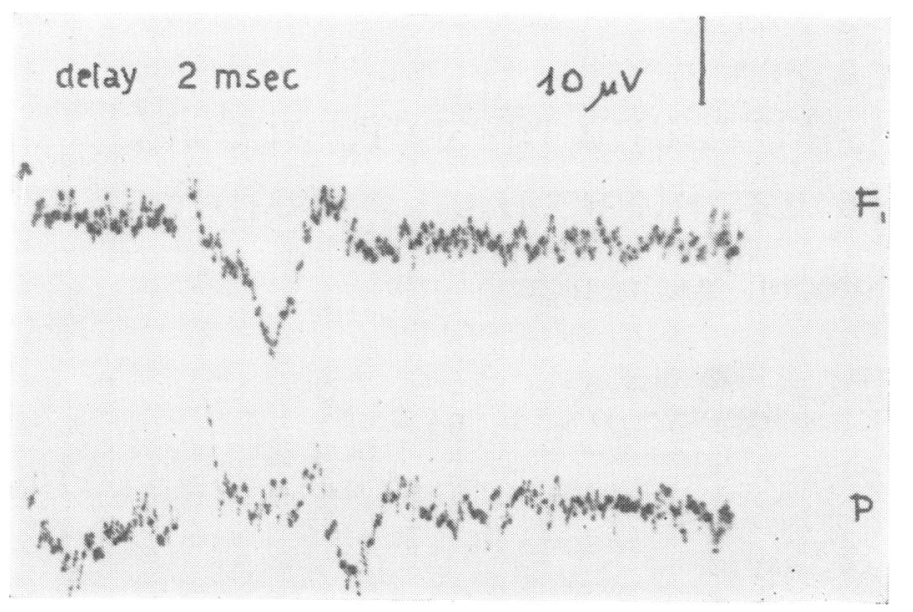

FIG. 7. An antidromic potential at the thumb and an orthodromic potential at the wrist were obtained at the same time by a stimulation of the sensory fibres at the palm (sweep. 1).

(1969) who carried out his studies also in patients with amyotrophic lateral sclerosis, when there had been complete loss of motor innervation, as well as in patients with plexus root avulsion, when the dorsal root ganglion cells were preserved with their axons. We do not believe that there is any contribution by motor axons; therefore we cannot agree with Trojaborg (1964) and Buchthal and Rosenfalck (1966).

The problem now is to explain the origin of this response. Our opinion is that this sensory antidromic response is derived by simple conduction from the larger sensory branches of the median nerve which derive from the thumb. In favour of this hypothesis is the fact that the prepotential was recorded with three electrodes placed over the sensory fibres to thenar muscles and the same latency was recorded in all three electrodes with progressive decreases in amplitude related to progressive distance from the sensory fibres.

Moreover, a stimulus applied to the palm evoked both an antidromic and an orthodromic response of equal threshold.

Finally, the pre-potential wave was recorded more easily with surface or wire-recording electrodes which pick up from a larger area than with the concentric needle.

The purpose of this study was to determine the origin of the pre-potential, a possible cause of error in motor latency measurements, especially when using high amplification. It could therefore be a technical error, seen mainly when using surface or wire recording electrodes. With time and more systematic investigation this phenomenon could be used as another parameter to test the abnormalities of large afferent nerve fibres. Lambert (1969) found this sensory potential reduced or absent in Friedreich's ataxia, but it was conducted with normal velocity in amyotrophic lateral sclerosis in spite of a slow motor nerve fibre conduction.

\section{REFERENCES}

Buchthal, F., and Rosenfalck, A. (1966). Evoked action potentials and conduction velocity in human sensory nerves. The intramuscular nerve action potential. Brain Research, 3, 72-73.

Dyck, P. J., and Lambert, E. H. (1968). Lower motor and primary sensory neuron diseases with peroneal muscular atrophy, 2. Archives of Neurology (Chic.), 18, 619-625.

Gutmann, L. (1969). The intramuscular nerve action potential. Journal of Neurology, Neurosurgery, and Psychiatry, 32, 193-196.

Lambert, E. H. (1969). Electromyography in amyotrophic lateral sclerosis. In Motor Neuron Diseases, pp. 135-153. Edited by F. H. Norris, Jr., and L. T. Kurland. Grune and Stratton: New York.

Simpson, J. A. (1964). Fact and fallacy in measurement of conduction velocity in motor nerves. Journal of Neurology, Neurosurgery, and Psychiatry, 27, 381-385.

Trojaborg, W. (1964). Motor nerve conduction velocities in normal subjects with particular reference to the conduction in proximal and distal segments of median and ulnar nerve. Electroencephalography and Clinical Neurophysiology, 17, 314-321. 